\title{
Towards the Next Generation of Information Systems: Enhancing Traceability and Transparency
}

\author{
Khin Than Win \\ School of Computing and IT, \\ University of Wollongong, \\ Wollongong \\ win@uow.edu.au
}

\section{Ghassan Beydoun}

School of Information, Systems and Modelling,

University of Technology Sydney,

Australia

\section{Abstract}

The information systems community plays a significant role in providing theories for guiding the creation of more effective information systems. This is nowadays most prominent in supporting the ongoing evolution of Artificial Intelligence (AI) and decision-making systems. As these systems are becoming increasingly reliable, and supplanting the presence of humans, the question of accountability and trust in these systems is increasingly coming to the fore. This editorial positions and showcases a collection of papers from the 2018 Australasian Conference on Information Systems within this context.

Keywords: Traceability, Decision Making, Transparency.

\section{Introduction}

Information Systems play a critical role in decision making processes for individuals and organisations. Individuals and organisations have adopted different tools to process and analyse data to assist in gaining useful information and knowledge. With the extreme growth of AI, deployment of intelligent systems within organisations seeking further automation is increasingly being pursued. However, an emerging barrier to the acceptance of intelligent information systems is their current inability to adequately explain their decisions. To equip such systems with the ability to explain and justify, it is clear that understanding the nature of explanations and what constitutes good human justifications and communication in a given context is critical. From this vantage point of providing explainability to enhance trust, it is becoming increasingly noted that social science is required to support the continuing evolution of intelligent systems (Miller 2019). The Information Systems community is best positioned to bridge social science to AI and IT. Hence, it is important that the IS community is further sensitized to this role/responsibility moving forward with AI and decision support systems.

In this short editorial paper, we first review the content of the section against the increasing importance of enhancing transparency and traceability of decision making in modern information systems. We further reflect on the role of the IS community in that endeavour and conclude with further remarks on what items an agenda for the next wave of IS efforts in this space could include. 


\section{Reviewing the content}

The contribution of IS to enhance decision transparency and traceability is expressed in different forms within the community. Many IS researchers are enhancing analytics tools and potentials by enhancing the role of visualization of data. This in turn translates further visibility into intelligent systems processes to gain insights into how they arrived at their decision. A good number of IS researchers are also concerned with modelling decision making in various applications areas, including the obvious area of information systems development itself. This collection of papers provides excellent exemplars of the breadth of Information Systems that can potentially bear on the challenge of enhancing trust and transparency of intelligent systems.

Four selected papers from ACIS (2018) presented information systems strategy in assisting decision-making. The best paper nominees from ACIS2018 were invited to expand their papers to the specifications of this journal and were accepted after two rounds of peer review and revisions. We thank all reviewers for their time spent on reviewing papers and providing feedback. The studies presented range from food safety and digital gaming to enterprise social networking adopting different information system theories.

The paper by Lam et al. (2020) presents the importance of information transparency in decision making related to food products. This work provides a compelling argument on how traceability can enhance trust in service delivery in the domain of food delivery. This paper concurs with the arguments presented in this preface and presents a good collection of IS related efforts to support the notion that traceability is essential to ensure accountability and trust. The results of the paper from a quantitative study confirms this position in a food supply chain example.

The paper by Reimer et al. (2020) provides results of activities in Enterprise Social Networks. They uncover hidden interaction patterns within the networks. By making these hidden patterns visible to management, they recommend their use to enable management to assess how the intent of the information system (i.e. the network) aligns with the outcome. This paper provides another powerful example of how uncovering hidden patterns and enhancing traceability of outcomes are critical to trust in the system.

The paper by Kautz et al. (2020) brings to the fore the importance of having well understood interactions in a distributed development environment. In other words, traceability of the development processes themselves is important to evaluate the success of the process. They present their studies in information system development in crowd sourcing using Complex Adaptive System theory. The study utilised Distributed Participatory Design in a digital game development.

The paper by Faber et al. (2020) tackles the challenges of visualizing business ecosystems to enable visibility and transparency of the underlying complex processes. The paper reports the results of two recent case studies by adopting an action design research approach. Again, this work addresses the key theme of this special issue in highlighting how traceability of the underlying processes in an information system is critical to trust its outcome.

\section{Concluding remarks}

This special section provides a forum for IS academics and practitioners to identify and share the challenges, opportunities, and solutions that improve the transparency and traceability in 
information systems. A particular focus is on the viewpoint of increased insights into data in complex settings. We seek to provide a forum to disseminate work aiming to enhance trust in systems from the perspective that accountability cannot be compromised as our reliance on information systems increases. This special section aims to enhance the awareness of the role of IS in that endeavour. IS perspectives include enhanced visualisations of data patterns, additional insights into the underlying processes and their interplay with the users as well as the development process of IT artefacts involved. Future efforts of the IS community along these three dimensions will continue to enhance the bridge between research undertaken in the social context of the system and research addressing their IT technological challenges.

\section{References}

Faber A., Hernandez-Mendez A., Rehm S-V, Matthes F. (2020), Collaborative Modelling and Visualization of Business Ecosystems: Insights from two Action Design Research Case Studies, Australasian Journal of Information Systems 24, https://doi.org/10.3127/ajis.v24i0.2229

Kautz K., Bjerknes G., Fisher J., Jensen T. (2020), Applying Complex Adaptive Systems Theory to Understand Distributed Participatory Design in Contemporary, Crowdsourced Information Systems Development, Australasian Journal of Information Systems 24 https://doi.org/10.3127/ajis.v24i0.2225

Lam T. K., Heales J., Hartley N., Hodkinson C. (2020), Consumer Trust in Food Safety Requires Information Transparency, Australasian Journal of Information Systems 24, https://doi.org/10.3127/ajis.v24i0.2219

Miller, (2019), Explanation in artificial intelligence: insights from the social sciences, Artificial intelligence 267, 1-38

Riemer K., Lee L.L., Kjaer C., Haeffner A. (2020), Identification of Enterprise Social Network (ESN) Group Archetypes in ESN Analytics - Metrics Selection and Case Application, Australasian Journal of Information Systems 24, https://doi.org/10.3127/ajis.v24i0.2355

Copyright: $@ 2020$ Win \& Beydoun. This is an open-access article distributed under the terms of the Creative Commons Attribution-NonCommercial 3.0 Australia License, which permits non-commercial use, distribution, and reproduction in any medium, provided the original author and AJIS are credited. 


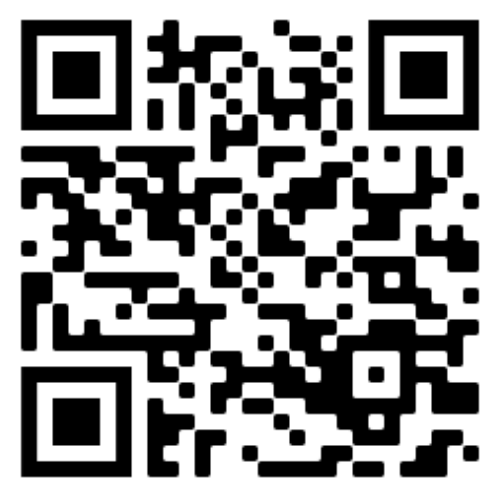

